\title{
A CMOS camera-based system for clinical photoplethysmographic applications
}

\author{
Kenneth Humphreys ${ }^{1}$, Charles Markham ${ }^{2}$, Tomás E. Ward ${ }^{1}$ \\ ${ }^{1}$ Department of Electronic Engineering, National University of Ireland, Maynooth, Ireland \\ ${ }^{2}$ Department of Computer Science, National University of Ireland, Maynooth, Ireland
}

\begin{abstract}
In this work an-image based photoplethysmography (PPG) system is developed and tested against a conventional fingerbased system as commonly used in clinical practise. A PPG is essentially an optical instrument consisting of a near infrared (NIR) source and detector that is capable of tracking blood flow changes in body tissue. When used with a number of wavelengths in the NIR band blood oxygenation changes as well as other blood chemical signatures can be ascertained yielding a very useful device in the clinical realm. Conventionally such a device requires direct contact with the tissue under investigation which eliminates the possibility of its use for applications like wound management where the tissue oxygenation measurement could be extremely useful. To circumnavigate this shortcoming we have developed a CMOS camera-based system, which can successfully extract the PPG signal without contact with the tissue under investigation. A comparison of our results with conventional techniques has yielded excellent results.
\end{abstract}

Keywords: Optical Tissue Imaging, near infrared spectroscopy, non-invasive physiological measurement, photoplethysmography, image processing.

\section{INTRODUCTION}

Photoplethysmography (PPG) is a near-infrared (NIR) measurement of blood flow in the body. A PPG is usually obtained by placing a probe on a subject's finger or earlobe and passing NIR light from a source to a detector, by either transmission or reflectance. Tissue is relatively transparent at NIR wavelengths, however haemoglobin - a respiratory protein found in red blood cells (erythrocytes) and responsible for oxygen transportation - absorbs NIR light strongly ${ }^{1}$. During the cardiac cycle at times of high pressure (systole), the combination of an increase in blood volume under the probe $^{2}$ and an alignment of the erythrocytes such that their long axis is perpendicular to the direction of blood flow ${ }^{3}$, cause an increase in light absorption. At low pressure (diastole), the blood volume under the probe decreases and the erythrocytes align their long axis parallel to the direction of blood flow, causing a decrease in light absorption. A PPG signal captures this activity. The value of a PPG signal in a clinical environment lies in its ability to provide a quick indication of the cardiac rhythm. The PPG signal's fine structure can also provide information about the condition of the vasculature, aortic valve as well as yield information on autonomic arousal ${ }^{4}$ and blood pressure autoregulation ${ }^{5}$.

Another important role of the PPG is in pulse oximetry ${ }^{6,7}$, the name given to the measuring of arterial oxygen saturation. Oxygen is transported around the body by forming reversible bonds with haemoglobin. The oxygenated $\left(\mathrm{HbO}_{2}\right)$ and deoxygenated $(\mathrm{Hb})$ forms of haemoglobin have different absorption spectra in the NIR band. By capturing a PPG at two suitable wavelengths the relative concentrations of $\mathrm{Hb}$ and $\mathrm{HbO}_{2}$ can be determined, from this the oxygen saturation can be extrapolated. Pulse oximetry can be used as an indicator of overall health, such as an early indicator of cyanosis during anaesthesia ${ }^{2}$, or as means of monitoring tissue viability, such as vascular injuries to limbs ${ }^{2}$, skin flaps elevated during plastic surgery ${ }^{8}$ or bowel viability'.

Current photoplethysmography and pulse oximetry devices require direct contact with tissue and record information from only one location at a time. There are several applications, such as wound management, where PPG and pulse oximetry in particular could provide useful insight, but where direct contact with tissue is undesirable. In these 
applications the ability to observe changes in oxygen saturation over an area without any contact with the tissue under investigation would be a great boon.

In this paper a CMOS camera-based PPG transmission imaging system is described and its performance is compared to a commercially available, conventional, direct-contact PPG probe, of the kind used in clinical practise. The use of a CMOS sensor represents a reduction in both the sensitivity and the cost of the equipment that has been applied to this task to date ${ }^{10}$. However the reduction in sensitivity has been compensated for by the use of a robust image processing technique, in addition the high frame rates achievable through CMOS sensor technology offer the possibility of extending PPG imaging to pulse oximetry imaging which is the ultimate goal of this research.

\section{METHODOLOGY}

Figure 1 illustrates the set-up for a transmission mode measurement of the PPG signal in an extremity (a finger is depicted in the figure although an earlobe would also be suitable). The subject's finger is illuminated on one side by a NIR light emitting diode (LED) (Opto Diode Corp. OD-1240) that emits light centred on a wavelength of $800 \mathrm{~nm}$, which is close to the isobestic point in the absorption spectra of $\mathrm{HbO}_{2}$ and $\mathrm{Hb}$ (where both compounds have the same light absorption). The LED is powered by a continuous $5 \mathrm{~V}$ DC supply. A $47 \Omega$ fixed resistor and a $0-100 \Omega$ potentiometer are placed in series with the LED. The diode draws a continuous current of 20-80 mA, which is varied depending on the subject. Under these conditions the diode emits a maximum power of $15 \mathrm{~mW}$. The camera used is a monochrome Pixelink PL-A741, which uses a 2/3" CMOS sensor with 1280x1024 square pixels of length $6.7 \mu \mathrm{m}$. Each pixel can be encoded using up to 10 bits. The camera connects to a computer via an IEEE 1394 (FireWire) connection. Videos are recorded to the computer in uncompressed AVI format using a progressive scan. Typically a sampling rate of 30 frames per second with an exposure time of $30 \mathrm{~ms}$ is sufficient to capture the fine structure of the PPG signal. The camera is focused on the finger using a C-mount Computar zoom lens (focal length $18 \mathrm{~mm}$ to $108 \mathrm{~mm}$, f2.5 to closed).

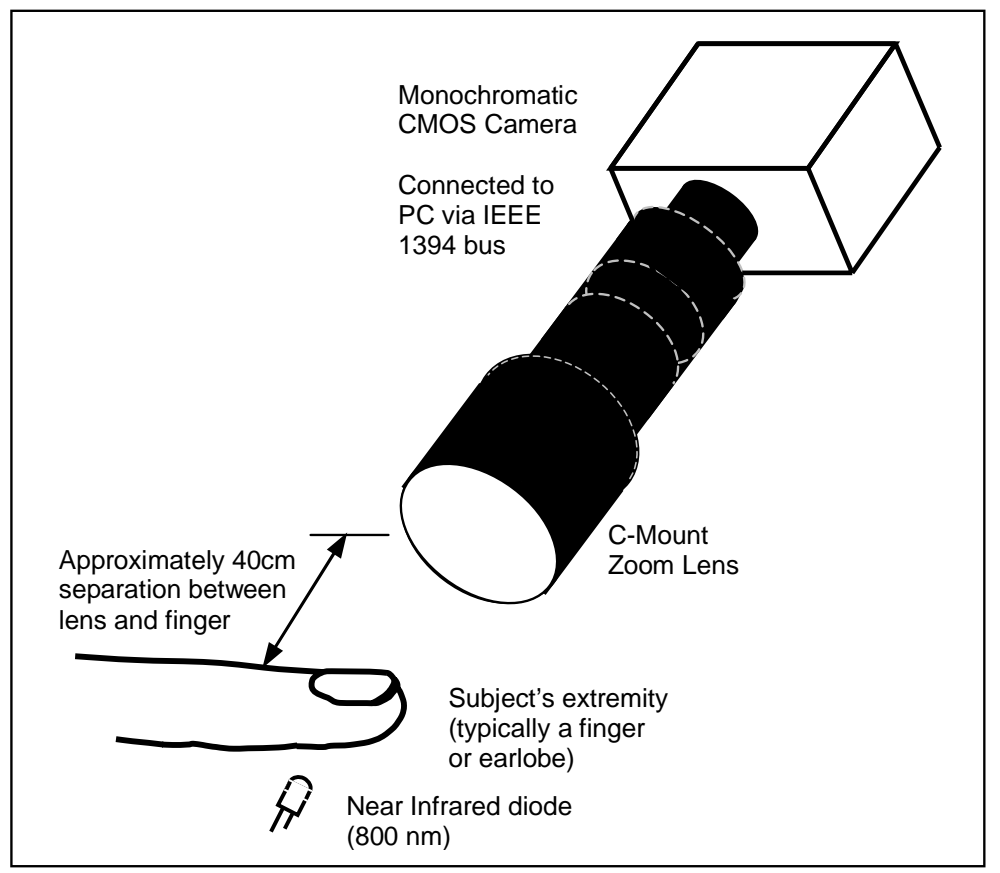

Figure 1: Transmission mode PPG imaging system. 
The camera is mounted on a tripod and positioned such that the lens face is between $30 \mathrm{~cm}$ and $50 \mathrm{~cm}$ from the subject's finger. Since it is required to view a relatively small area - only a few square centimetres - the camera's full spatial resolution of 1.3 million pixels is not needed. Selecting an area in the centre of the CMOS sensor measuring $240 \times 320$ pixels provides more than adequate spatial resolution and allows for significantly higher frame rates. The recorded AVI format videos are processed using Matlab®. Each video frame consisting of $240 \times 320$ pixels is divided into several boxes or groups of adjacent pixels. The average value of the pixels in each box is calculated for each frame. This is effectively a measure of how the light intensity in a particular part of the frame varies from frame to frame. Since the change in light intensity on the surface of the finger is due at least in part to the pulsing of the blood through the finger, a PPG signal is evident in the video image sequences. The process is illustrated in Figure 2. The change in the average pixel value of a box measuring 20x20 pixels (outlined in white in Figure 2) is plotted against time. Naturally the pulsing blood is not the only source of variation in light intensity - no NIR filter is used and the CMOS sensor is more sensitive to visible light than NIR light - artificial room lighting as well as sunlight affect the light intensity registered by the sensor. Despite this a clear arterial pressure waveform is visible in the plot without performing any digital filtering or signal processing. As stated earlier an increase in light intensity actually corresponds to a decrease in blood pressure, thus plotting the average pixel value multiplied by minus one yields the familiar pulse shape as shown in Figure 2 (B).

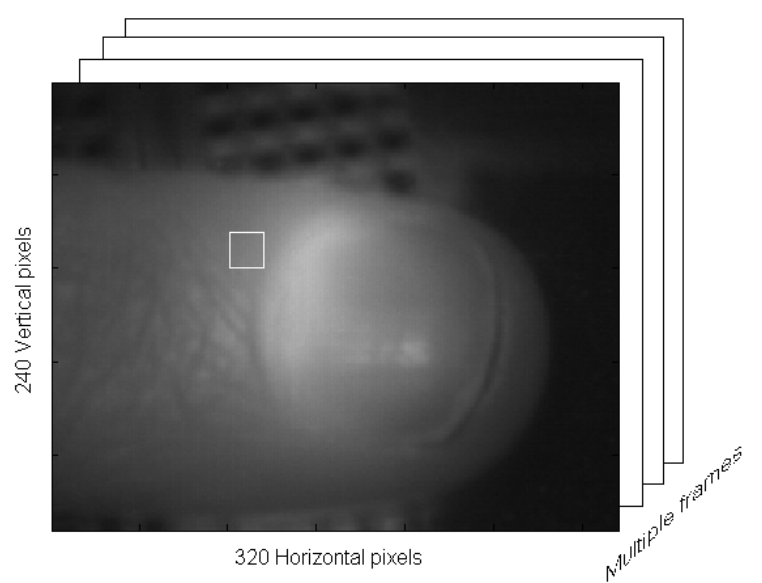

(A)

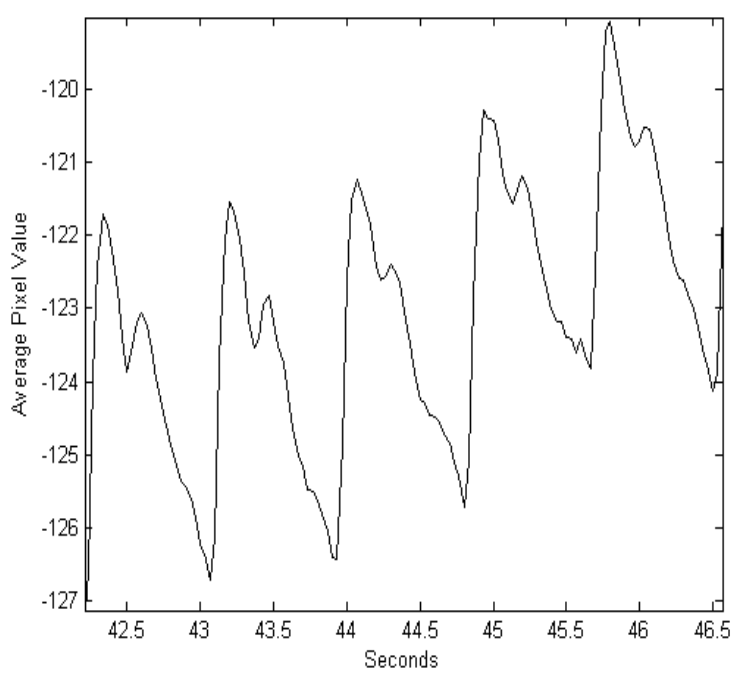

(B)

Figure 2: (A) A group of 400 pixels whose average value is calculated once each frame and (B) how that average value changes over time.

Clearly not every part of the video will contain a PPG signal - indeed a portion of the pixels are not focused on the subject's finger at all. Of the boxes in each frame that contain a PPG signal, some are more suitable than others, depending on the amount of illumination and blood an area receives. It is desirable to be able to quickly identify the boxes that contain a good signal. A simple way of doing this is to count the number of inflection points in the signal during a suitable period of time. A PPG signal can vary considerably in appearance depending on the age and health of the subject, however regardless of appearance the signal should contain no more than 4 points of inflection per heartbeat. A noisy signal will likely contain more than 4 points of inflection over the duration of a heartbeat and can be eliminated from the search on this basis. The inflection points are identified as follows:

1. A segment corresponding to one second of a waveform is isolated.

2. Differencing successive samples as described by (1) forms an approximate derivative of that segment. 


$$
x^{\prime}[n]=x[n+1]-x[n]
$$

3. A new vector, $S$, is assigned the signs (+/-) of the samples of the differentiated segment.

4. The vector $S$ is right shifted by one sample to create the vector $\hat{S}$.

5. The vectors $S$ and $\hat{S}$ are compared - any place where they do not have the same sign represents an inflection point at that place in the vector $S$ - the number of these inflection points are counted.

6. Steps $2-4$ are repeated for the next three one-second segments of the waveform.

7. If the mean number of inflection points over four segments is less than a threshold value, say 6 points, that area of the video is deemed to contain a PPG signal.

8. If too few viable PPG signals are identified the threshold value is increased and the process begins again.

It is worth noting that areas of a video that do not meet this criterion may do so after signal processing has been applied.

\section{RESULTS}

A comparison was made between the performance of the camera-based system and conventional, direct-contact, PPG sensor. A Biopac Systems Inc. PPG 100C module with a gain of 100 and band-pass filter with a pass band from 0.05 $\mathrm{Hz}$ to $10 \mathrm{~Hz}$ was used in conjunction with an Xdcr Tp PPG sensor. This is a reflection mode sensor consisting of one LED source and one photodiode detector side by side. The device is secured to a subject's finger by means of an elastic Velcro strap. This device is typical of those used in clinical practise.

To perform the comparison of the two systems, experiments were performed on seated subjects with each subject's forearm resting on a table. The conventional contact Biopac PPG sensor was secured to the tip of either the subjects' middle or index finger while an NIR LED illuminated the underside of their adjacent (middle or index) finger. The camera was then focused on the illuminated finger and PPG signals from both fingers were captured simultaneously over a period of one minute.

The plots in Figure 3 below show results obtained from a male subject aged 25. The direct-contact and camera-based systems recorded the PPG signals at 30 samples and 30 frames per second respectively. Each frame of the PPG video was processed in boxes of $20 \times 20$ pixels. The camera PPG shown in Figure 3 was chosen at random from all 20x20 pixel areas of the video deemed to contain a good PPG signal based on the inflection point criterion described above. 


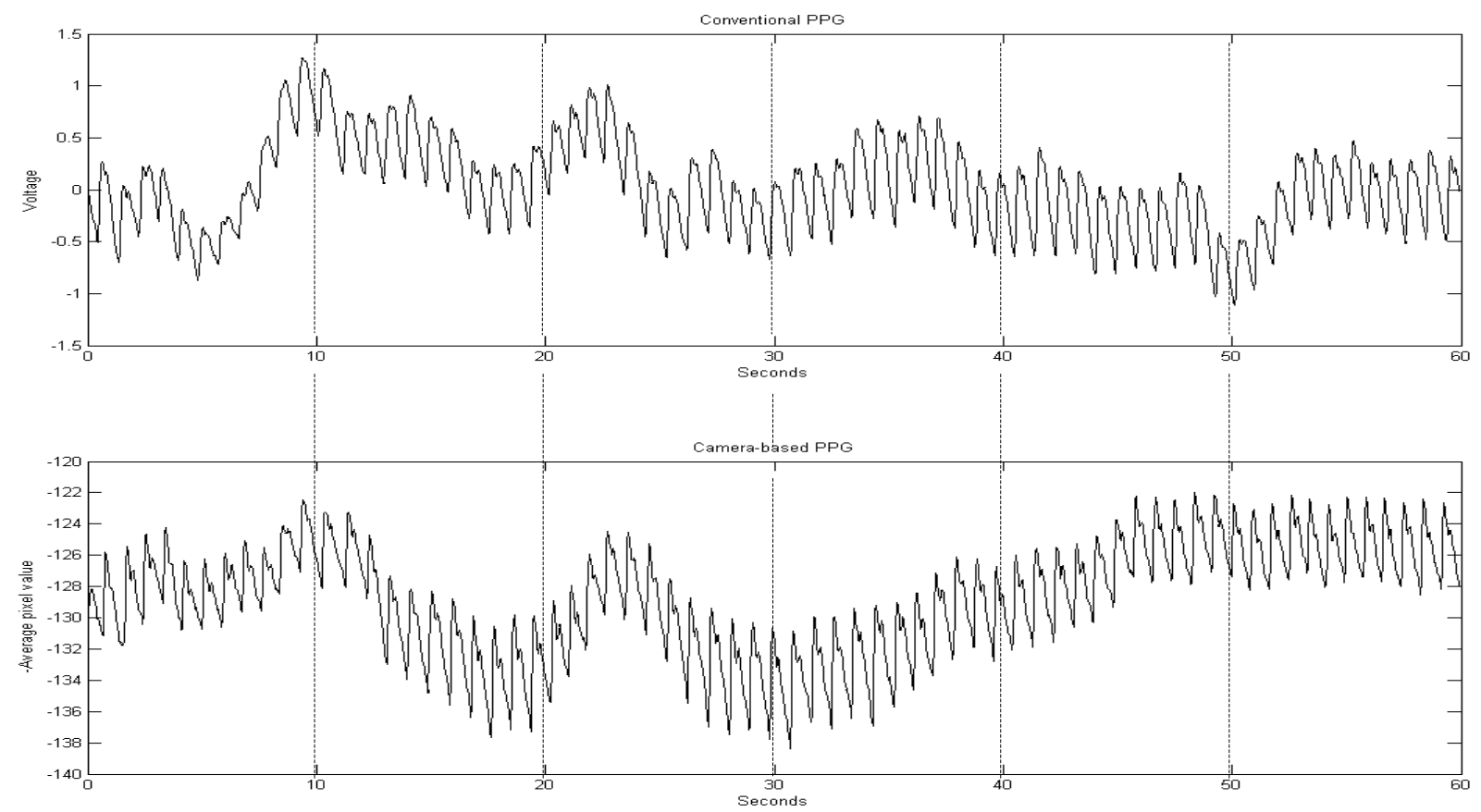

Figure 3: Comparison of the PPG signals obtained using a conventional (top) PPG sensor and a camera-based (bottom) system.

It can be seen in Figure 3 that the PPG signals obtained using the camera-based system is similar to the conventionally obtained signal. Both signals show the same number of well-defined heartbeats and both signals also show slower oscillations typical of the blood pressure waveform ${ }^{11}$. Since the conventional PPG sensor is relatively well shielded from changes in ambient light, the slower oscillations in this signal can be largely attributed to biological activities that affect blood flow, primarily breathing. The camera-based system, being much more susceptible to ambient light changes, shows a slightly different pattern of low-frequency oscillations.

In order to compare the fine structure of the PPG signals, both signals were linearly de-trended. The low frequency oscillations are generally too great to achieve a good-fit single-line linear de-trend. To compensate for this, de-trending was performed on a piece-wise basis, by splitting both signals into contiguous frames of 30 samples (1 second) and subtracting the mean value from each frame. In addition, since the Biopac instrument employs a band-pass filter in capturing the conventional PPG signal, a low-pass filter was applied to the camera PPG. The transfer function of that filter is described by equation (2).

$$
H(Z)=\frac{1-Z^{-1}}{1-0.9880 Z^{-1}}
$$

At a sampling rate of $30 \mathrm{~Hz}$, this filter has a $-3 \mathrm{~dB}$ cut-off frequency of $0.056 \mathrm{~Hz}$, which is almost equal to the $0.05 \mathrm{~Hz}$ lower cut-off applied by the Biopac PPG module. 
Figure 4 displays a comparison of the conventional and camera-based PPG signals (shown in Figure 3) after both have been filtered and de-trended.
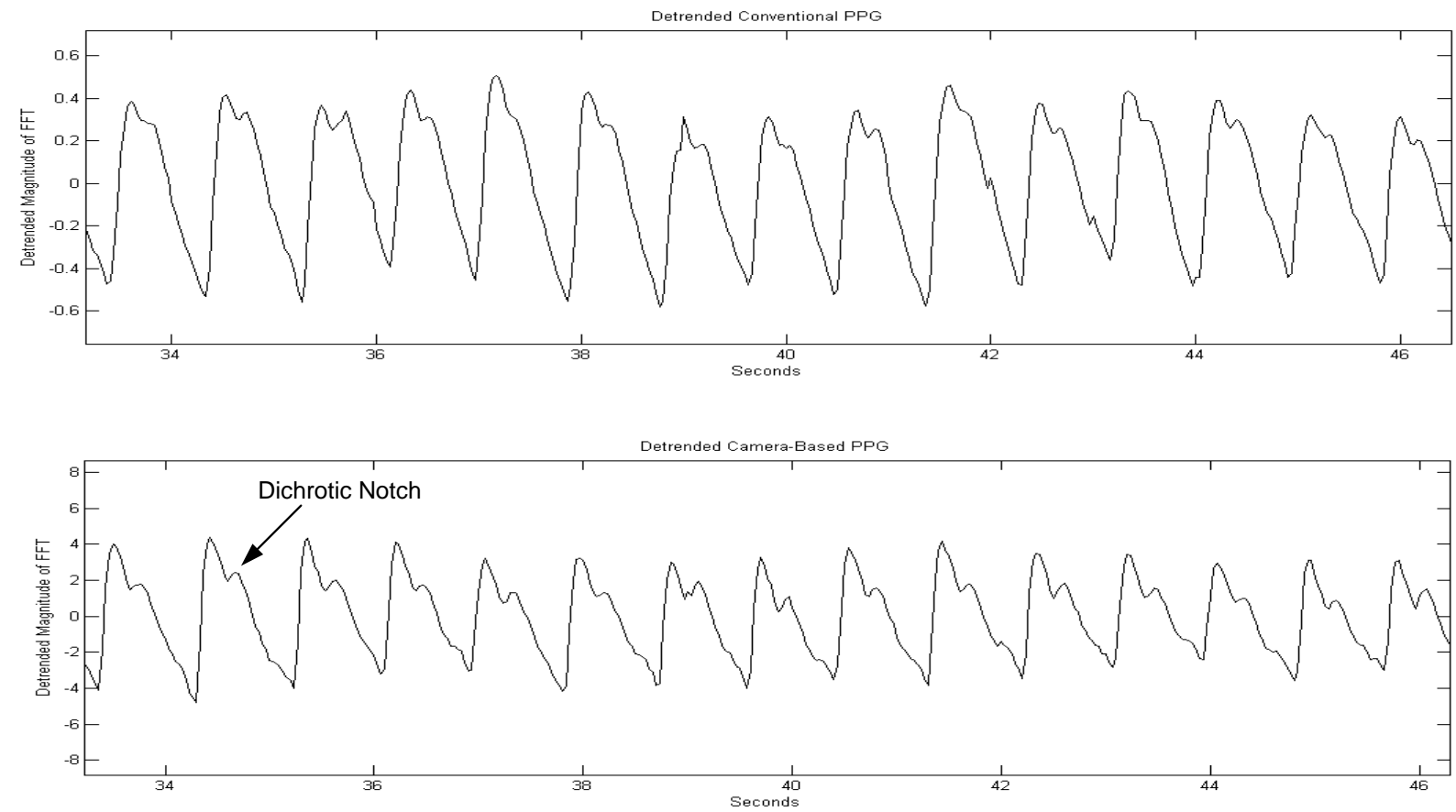

Figure 4: Comparison of the de-trended PPG signals obtained using a conventional PPG sensor (top) and a camera-based system (bottom).

It can be seen in Figure 4 that the fine structure of the PPG, in particular the dichrotic notch - the brief backflow of blood associated with the closure of the aortic valve - is more pronounced and consistently visible in the camera-based signal. In order to ensure that the difference in the fine structure of the signals is due to the performance of the two systems, rather than a result of measuring the signal on two different fingers, an experiment was performed in which two adjacent fingers were illuminated by two separate but identical diodes, and a video was made with both illuminated fingers in view. In this way two PPG signals were captured simultaneously using only the camera-based system. The results in of this experiment are shown in Figure 5. Figure 5 (A) is a sample frame from the video, in which the black squares indicate $20 \times 20$ pixel areas of the frame where PPG signals were found using the inflection point criterion. Figure 5 (B) depicts sample PPG signals from the index finger and the middle finger visible in the top and bottom of Figure 5 (A) respectively. It can be seen in Figure 5 that although the PPG signals differ in appearance, the characteristic shape and dichrotic notch is clearly visible in both. 


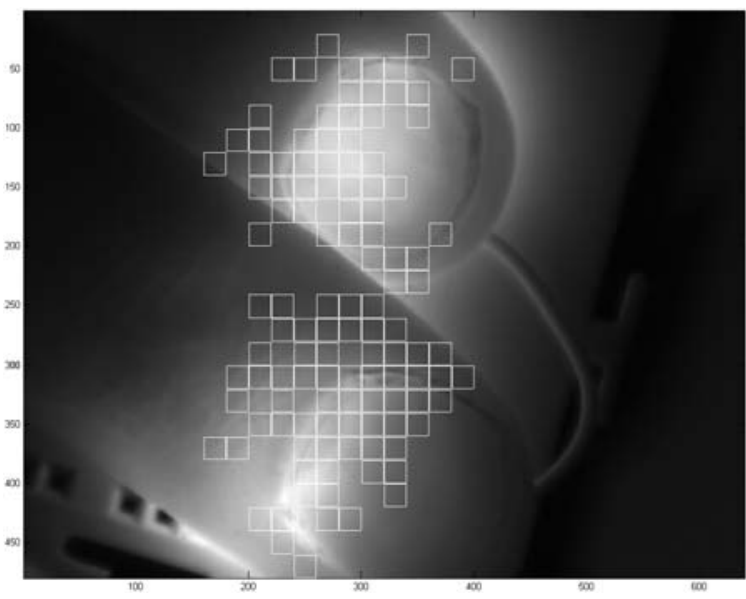

(A)

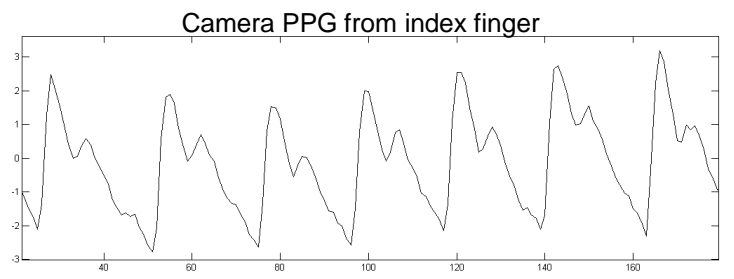

Camera PPG from middle finger

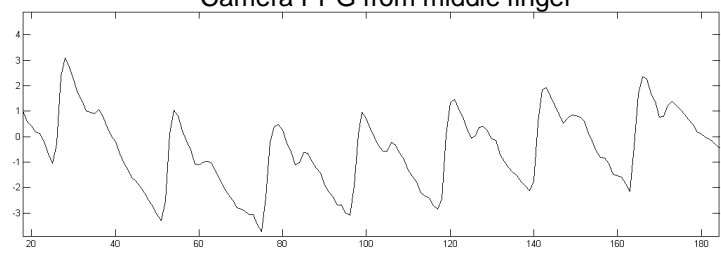

(B)

Figure 5: (A) A sample video frame with 20x20 pixel boxes imposed on it to indicate the locations of PPG signals that met the inflection point criterion described earlier. (B) PPG signals from each of the fingertips visible in (A).

Figure 6 shows the frequency spectra associated with the time domain plots in Figure 3. Again both time-domain signals have been de-trended prior to calculating their spectra. Upon comparing the two spectra the heart rate is immediately apparent in both signals at approximately $1.16 \mathrm{~Hz}$. The lower frequency vasomotion and Mayer wave can be seen well in both signals from approximately $0.1 \mathrm{~Hz}$ and downward ${ }^{12}$. The breathing rate is apparent at approximately $0.25 \mathrm{~Hz}$ in the conventional PPG signal, but is less obvious in the camera-based PPG.
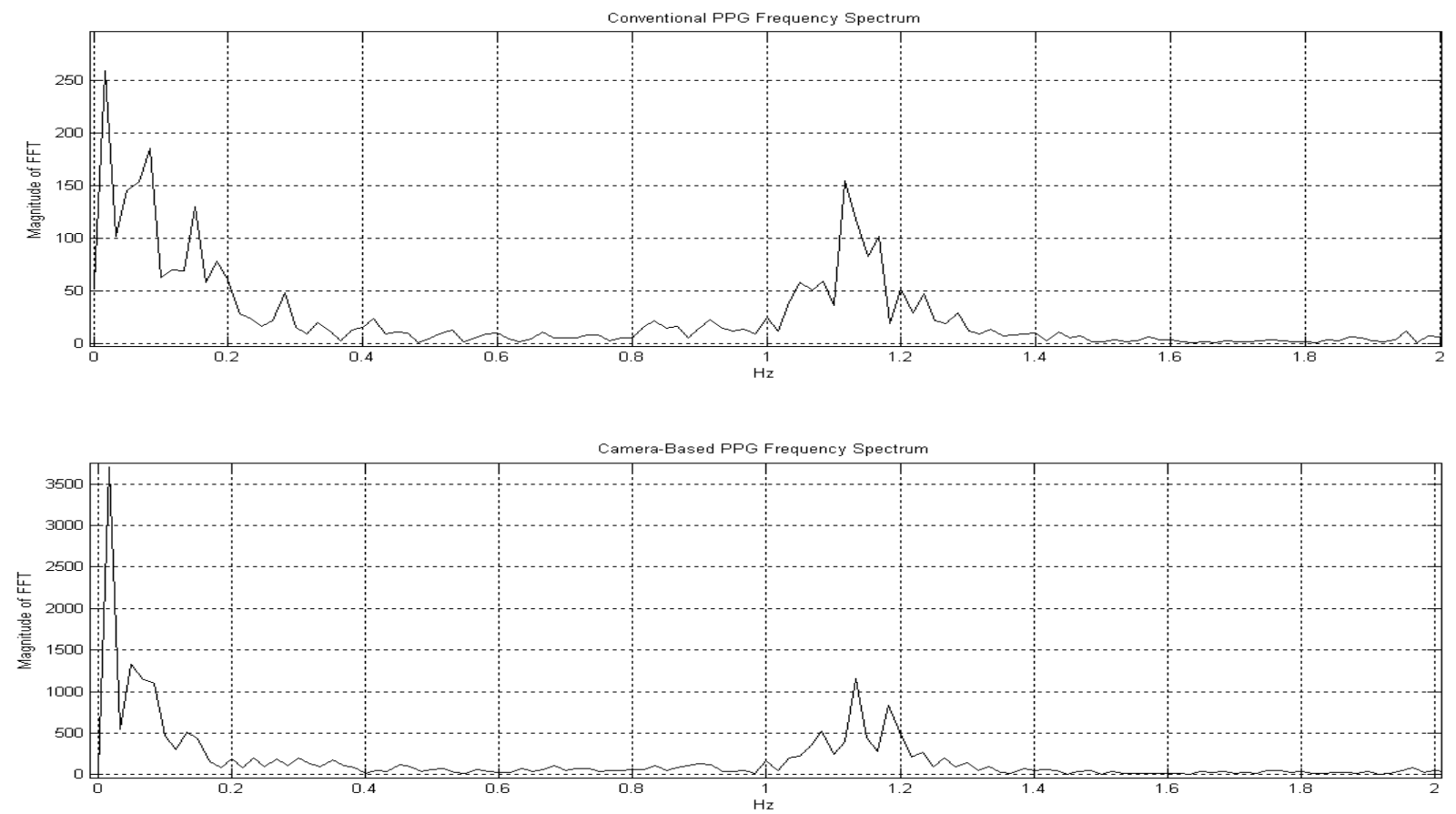

Figure 6: Comparison of the frequency spectra of a conventional PPG sensor (top) and a camera-based system (bottom). 


\section{DISCUSSION}

The energy of a photon at the far end of the NIR band (around $900 \mathrm{~nm}$ ) is approximately $1.3 \mathrm{eV}$, little more than the 1.1 $\mathrm{eV}$ required to break Silicon's covalent bonds and register the incoming light. It is not uncommon then to encounter specialised cooled and back-illuminated charge coupled device (CCD) sensors being used in NIR spectroscopic imaging applications $8,10,13,14$. CMOS sensors owing to their inherently lower pixel fill-factor offer less quantum efficiency than their CCD counterpart and are rarely encountered in applications at this wavelength. The Pixelink camera used in this application is primarily intended for machine-vision applications. Across the NIR band it has a sensitivity of the order $0.085 \mathrm{~A} / \mathrm{W}$ at $740 \mathrm{~nm}$ to $0.028 \mathrm{~A} / \mathrm{W}$ at $900 \mathrm{~nm}$, a quantum efficiency of approximately $14 \%$ to $3 \%$. Even so it has been shown to be capable of the task. CMOS sensors offer two principal advantages over CCD sensors, they permit higher frame rates and they are cheaper to manufacture and buy. In particular the high frame rates offered by CMOD can be exploited to acquire time-multiplexed PPG signals at multiple wavelengths, offering the possibility of inexpensive realtime tissue oxygenation imaging. Such a device could have considerable applications in non-invasive wound assessment such as tissue viability measurement for burns patients.

\section{REFERENCES}

1. $\quad$ Elwell C., A practical users guide to near infrared spectroscopy. 1995: Hamamatsu Photonics KK.

2. Moyle J., Pulse Oximitery. 1994: BMJ Publishing Group.

3. Nijboer J., Dorlas J., and Mahieu H., Photoelectric plethysmography - some fundamental aspects of the reflection and transmission method, Clinical Physics and Physiological Measurement, 7, 205-215, 1981.

4. Coyle S., Ward T., Markham C., Roche B., McDarby G., and McLoone S. The use of near-infrared spectroscopy in measuring general autonomic arousal. in World Congress on Medical Physics and Biomedical Engineering. 2003. Sydney, Australia.

5. Kinanne O., Ph.D. Thesis, Dept. of Electronic Engineering, National University of Ireland, 2005

6. Kelleher J., Pulse oximetry, Journal of Clinical Monitoring, 5, 37-62, 1989.

7. $\quad$ Payne J. and Severinghaus J., Pulse oximetry. 1986: Springer, New York.

8. Attas M., Hewko M., Pologe J., Posthumus T., Sowa M., and Mantsch H., Visualization of cutaneous hemoglobin oxygenation and skin hydration using near-infrared spectroscopic imaging, Skin Research and Technology, 7, (4), 238-245, 2001.

9. DeNobile J., Guzzetta P., and Patterson K., Pulse oximetry as a means of assessing bowel viability, Journal of Surgical Research, 48, 21-23, 1990.

10. Blazek V., Wu T., and Holscher D., Near-infrared CCD imaging - possibilities for noninvasive and contactless $2 D$ mapping of dermal venous hemodynamics, Proceedings of SPIE, 3923, 2-9, 2000.

11. Guyton A. and Hall E., Human Physiology and Mechanics of Disease. 4 ed. 1987: W.B. Saunders.

12. Elwell C., Springett R., Hillman E., and Delpy D., Oscillations in cerebral haemodynamics, Advances in experimental medicine and biology, 471, 57-65, 1999.

13. Sowa M., Payette J., Hewko M., and Mantsch H., Visible near infrared multispectral imaging of the rat dorsal skin flap, Journal of Biomedical Optics, 4, (4), 474-481, 1999.

14. Hulsbusch M. and Blazek V., Contactless mapping of rhythmical phenomena in tissue perfusion using PPGI, Proceedings of SPIE, 4683, 110-117, 2002. 